\title{
Scrub Typhus and Abnormal Electrocardiography
}

\author{
Seo-Won Choi, ${ }^{1} \mathrm{Na}$ Ra Yun, ${ }^{1}$ Dong-Hyun Choi, ${ }^{1}$ Young-Jae Ki, ${ }^{1}$ Seok Won Kim, ${ }^{2}$ Choon-Mee Kim, ${ }^{3}$ and Dong-Min Kim ${ }^{1 *}$ \\ ${ }^{1}$ Department of Internal Medicine, School of Medicine, Chosun University, Gwangju, Republic of Korea; ${ }^{2}$ Department of Neurosurgery, \\ School of Medicine, Chosun University, Gwangju, Republic of Korea; ${ }^{3}$ The Division of Natural Medical Sciences, College of Health Science, \\ Chosun University, Gwangju, Republic of Korea
}

\begin{abstract}
This study compared the frequency of abnormal electrocardiogram (ECG) types between scrub typhus patient group and age- and gender-matched health checkup group and their associations with disease severity in scrub typhus patient. Demographic characteristics and ECG and laboratory findings of patients with scrub typhus admitted to Chosun University Hospital, and normal subjects visiting the hospital for health checkup from January 2008 to December 2012 were retrospectively studied. Electrocardiogram abnormalities at admission were observed in 72 of 165 (43.6\%) scrub typhus confirmed patients. The following ECG abnormalities were observed: arrhythmic group (31 cases, $18.8 \%)$, ischemic change group (25 cases, 15.1\%), prolonged QT group (32 cases, 19.4\%).Compared with the age and gendermatched health checkup group, ECG abnormalities were more commonly observed in scrub typhus patient group (13.9\% versus $43.6 \%, P<0.001$ ). In addition, when compared with the normal ECG group, scrub typhus in the abnormal ECG group showed greater disease severity and this phenomenon was particularly prominent in the prolonged QT group. Based on our study prolonged QT observed in approximately $20 \%$ of patients with scrub typhus, clinicians should pay additional attention to drugs that affect QT interval.
\end{abstract}

\section{INTRODUCTION}

Scrub typhus is an acute febrile disease characterized by systemic vasculitis that is transmitted via bites from mite larvae (chigger) infected with Orientia tsutsugamushi. ${ }^{1}$ The incidence of scrub typhus is generally high in Asia, although recent outbreaks have been reported in Western countries along with the increase in international travel. ${ }^{2,3}$ Scrub typhus can be cured with antibiotics, if the clinical disease course is not severe, although a delayed diagnosis may lead to severe complications such as pneumonia, acute kidney injury, meningitis, encephalitis, upper gastrointestinal bleeding, and multiple organ failure, as well as the possibility of myocardial infarction or stroke, and some patients may die from such complications. $^{4}$

Most patients with scrub typhus is known to be shown with normal electrocardiogram (ECG) findings, although some patients present with nonspecific ECG changes such as ST segment changes, $T$ wave changes, and atrial premature beat (APB) and ventricular premature beat (VPB). On one hand, severe ECG changes such as atrial fibrillation (AFib), atrial flutter, and torsades de pointes have also been reported. ${ }^{5}$ However, few studies have evaluated ECG abnormalities in scrub typhus patients.

Accordingly, this study aimed to confirm the frequency of ECG abnormalities and commonly observed ECG type in the scrub typhus patients. We also tried to check the differences between the scrub typhus patients and the general population in the aspect of ECG abnormality and ascertain the relationship between ECG abnormalities and disease severity.

\section{MATERIALS AND METHODS}

Among 253 patients with scrub typhus hospitalized at Chosun University Hospital during the 5-year period from January 2008 to December 2012, 88 patients with missing

\footnotetext{
*Address correspondence to Dong-Min Kim, Department of Internal Medicine, College of Medicine, Chosun University, 588 Seosuk-dong, Dong-gu, Gwangju 501-717, Republic of Korea. E-mail: drongkim@ chosun.ac.kr
}

ECG records were excluded; thus, a total of 165 patients were retrospectively investigated in this study. Subjects who visited Chosun University Hospital during the same period for the purpose of health checkups served as age- and gendermatched controls at a 1:1 ratio.

The patients with scrub typhus were defined as those admitted to the hospital with characteristic eschar and typical clinical symptoms such as fever, chills, headache, and skin rash. Scrub typhus patients also exhibited $>4$-fold increases in antibody titers on indirect immunofluorescent antibody tests during the recovery period or received positive diagnoses via nested polymerase chain reaction tests using material obtained from buffy coats or eschar samples.

The patients' age, gender, and underlying health factors, including cigarette smoking, hypertension (HTN), diabetes mellitus (DM), cardiovascular disease, pulmonary disease, hepatic disease, and malignancy were investigated.

The patients with a normal sinus rhythm (NSR), including sinus tachycardia during ECG at admission were classified as the normal ECG group, whereas those with ECG findings other than NSR were classified as the abnormal ECG group. The latter group was further subclassified as follows: the arrhythmia group represented with Afib, APB, VPB, and conduction delay, such as atrioventricular block, right bundle branch block, and left bundle branch block; the ischemic group represented with ST-segment changes, $\mathrm{T}$ wave changes, and abnormal $Q$ waves; and the prolonged QT group.

A 12-lead ECG was obtained using a MAC 5500 machine (GE Healthcare, Little Chalfont, United Kingdom), and ECG records were classified using a modified version of the Minnesota code (Table 1). ${ }^{6}$

To evaluate scrub typhus severity, this study defined the patients with the following complications such as interstitial pneumonia, myocarditis, meningitis, acute respiratory distress, acute renal failure, shock, gastrointestinal bleeding, and death as serious patients.

Statistical analysis was performed using SPSS for Windows version 18.0 (SPSS, Inc., Chicago, IL). Continuous variables were described as mean values \pm standard deviations, and noncontinuous variables were described as frequencies and percentages (\%). Subject comparisons were performed using 
TABLE 1

Definition of abnormal electrocardiogram finding according to the modified version of the Minnesota code

\begin{tabular}{|c|c|}
\hline Q wave & $Q / R \geq 1 / 5, Q$ duration $\geq 0.03$ seconds $(s)$ \\
\hline ST segment & ST depression $\geq 1 \mathrm{~mm}(\mathrm{I}, \mathrm{II}, \mathrm{aVL}, \mathrm{aVF}, \mathrm{V} 1-6)$ \\
\hline T wave & $\begin{array}{l}\text { T wave } \geq-5 \mathrm{~mm}(\mathrm{I}, \mathrm{II}, \mathrm{V} 2-6) \text {, when } \mathrm{R} \geq 5 \mathrm{~mm} \\
\text { (aVL) and QRS upright (aVF) }\end{array}$ \\
\hline AV conduction & $\begin{array}{l}\mathrm{RP}>0.21 \text { seconds (I, II, III), partial AV } \\
\text { block, complete AV block }\end{array}$ \\
\hline Ventricular conduction & QRS $>0.12$ seconds \\
\hline Atrial fibrillation & Atrial fibrillation present \\
\hline Atrial flutter & Atrial flutter present \\
\hline Premature beat & $\begin{array}{l}\text { Atrial premature beat and ventricular } \\
\text { premature beat present }\end{array}$ \\
\hline Poor R progression & $\begin{array}{l}R \text { wave }<2-4 \mathrm{~mm} \text { in } \mathrm{V} 3 \text { or } \mathrm{V} 4 \\
\mathrm{R} \text { in } \mathrm{V} 4<\mathrm{R} \text { in } \mathrm{V} 3, \mathrm{R} \text { in } \mathrm{V} 3<\mathrm{R} \text { in } \mathrm{V} 2, \mathrm{R} \text { in } \mathrm{V} 2 \\
\quad<\mathrm{R} \text { in } \mathrm{V} 1\end{array}$ \\
\hline Long QT interval ${ }^{22}$ & $\begin{array}{l}\text { Corrected QT interval } \\
\text { Male }>0.45 \text { seconds, Female }>0.46 \\
\text { seconds }\end{array}$ \\
\hline
\end{tabular}

one-way analysis of variance and the $x^{2}$ test. The significance level was set at $P<0.05$.

\section{RESULTS}

Patient characteristics. The subjects included a total of 165 patients with scrub typhus (after excluding 88 patients with missing ECG records) who were hospitalized for scrub typhus at Chosun University Hospital during the study period. The mean age was $63.3 \pm 13.3$ years, and the subjects included 58 men (35.2\%) and 19 cigarette smokers (11.5\%). The underlying diseases included 56 cases (33.9\%) of HTN, 31 cases $(18.8 \%)$ of DM, four cases $(2.4 \%)$ of cardiovascular disease, three cases $(1.8 \%)$ of pulmonary disease, 14 cases (14.9\%) of hepatic disease, and eight cases (4.8\%) of malignancy.

The frequency of ECG abnormality at admission in patients with scrub typhus. At admission, ECG abnormalities were observed in 72 (43.6\%) of 165 patients with scrub typhus; the remaining 93 patients (56.4\%) with scrub typhus showed normal ECG results, including 26 patients with sinus tachycardia. The following ECG abnormalities were observed: arrhythmia group (31 cases, 18.8\%); ischemic change group (25 cases, 15.1\%); and prolonged QT group (32 cases, 19.4\%) (Table 2).

TABLE 2

ECG abnormality in 165 scrub typhus patients

\begin{tabular}{lc}
\hline ECG abnormalities & $72(43.6 \%)$ \\
Arrhythmic group & $31(18.8 \%)$ \\
Atrial fibrillation & $15(9.1 \%)$ \\
Atrial premature beat & $5(3.0 \%)$ \\
Ventricular premature beat & $1(0.6 \%)$ \\
$1^{\circ} \mathrm{AV}$ block & $6(3.6 \%)$ \\
$2^{\circ} \mathrm{AV}$ block & $0(0 \%)$ \\
$3^{\circ} \mathrm{AV}$ block & $1(0.6 \%)$ \\
Right bundle branch block & $5(3.0 \%)$ \\
Left bundle branch block & $1(0.6 \%)$ \\
Ischemic change group & $25(15.1 \%)$ \\
ST depression & $0(0 \%)$ \\
ST elevation & $1(0.6 \%)$ \\
T wave inversion & $21(12.7 \%)$ \\
Q wave & $3(1.8 \%)$ \\
Poor R progression & $3(1.8 \%)$ \\
Prolonged QT group & $32(19.4 \%)$ \\
\hline
\end{tabular}

Cf $>\mathrm{AV}=$ atrioventricular; $\mathrm{ECG}=$ electrocardiogram
Comparison of ECG abnormalities between the health checkup group and patients with scrub typhus. A total of 165 patients with scrub typhus were compared with 165 control subjects who visited Chosun University Hospital for health checkup during the study period and were age- and gender-matched with the scrub typhus patients at a 1:1 ratio. Electrocardiogram abnormalities were more common in the patient group than in the health checkup group (43.6\% versus $13.9 \%, P<0.001$ ), particularly in the prolonged QT group (19.4\% versus $2.4 \%, P<0.001)$, ischemic change group (15.2\% versus $7.9 \%, P=0.039$ ) (Table 3 ). Regarding laboratory findings, compared with the health checkup group, the scrub typhus patient group showed increased white blood cell counts (WBC; 8,241 \pm 4,036/L versus 6,024 $\pm 1,912 / \mathrm{L}$, $P<0.001)$, aspartate transaminase levels $(160 \pm 460 \mathrm{U} / \mathrm{L}$ versus $26 \pm 12 \mathrm{U} / \mathrm{L}, P<0.001$ ), alanine transaminase levels (92 $\pm 108 \mathrm{U} / \mathrm{L}$ versus $23 \pm 15 \mathrm{U} / \mathrm{L}, P<0.001)$, blood urea nitrogen levels (BUN; $18.1 \pm 12.9 \mathrm{mg} / \mathrm{dL}$ versus $15.08 \pm 4.0 \mathrm{mg} / \mathrm{dL}$, $P=0.004)$, total bilirubin levels $(0.92 \pm 0.62 \mathrm{mg} / \mathrm{dL}$ versus $0.78 \pm$ $0.27 \mathrm{mg} / \mathrm{dL}, P=0.008)$, and creatinine levels $(1.14 \pm 0.64 \mathrm{mg} / \mathrm{dL}$ versus $1.00 \pm 0.16 \mathrm{mg} / \mathrm{dL}, P=0.006)$ and significantly lower platelet counts $\left(170 \pm 91 \times 10^{6} / \mathrm{L}\right.$ versus $\left.261 \pm 64 \times 10^{6} / \mathrm{L}, P<0.001\right)$ and hemoglobin levels $(12.6 \pm 1.7 \mathrm{~g} / \mathrm{dL}$ versus $13.6 \pm 1.2 \mathrm{~g} / \mathrm{dL}$, $P<0.001$ ).

Comparison among patients with scrub typhus according to the presence of ECG abnormalities at admission. The patients with scrub typhus in the abnormal ECG group (based on ECG findings at admission) were significantly older than those in the normal ECG group (66.2 years versus 61.1 years, $P=0.014)$ and had higher prevalence of HTN (43.1\% versus $26.9 \%, P=0.03$ ); no significant differences in gender and underlying disease were observed between the groups.

Regarding the laboratory findings, the abnormal ECG group showed increased WBC counts, total bilirubin, BUN, and creatinine level (Table 4). Regarding the disease severity of scrub typhus patients, the abnormal ECG group showed greater disease severity compared with normal ECG group (34.7\% versus $20.4 \%, P=0.04$, Table 4 ).

Comparison between the normal ECG group and abnormal ECG subgroups at admission in patients with scrub typhus. In a comparison of the abnormal ECG subgroup with the normal ECG group, arrhythmia group patients were older, had a higher prevalence rate of HTN, DM and showed increased WBC, BUN, creatinine, total bilirubin, and lactate dehydrogenase; these arrhythmia group patients also showed lower levels of albumin, sodium ( $\mathrm{Na})$, chloride $(\mathrm{Cl})$, and calcium (Ca) (Table 5).

Compared with the normal ECG group, ischemia group revealed no significant differences. However, prolonged QT group defined by corrected QT interval prolongation values $>0.45$ milliseconds $(\mathrm{ms})$ in men and $>0.46 \mathrm{~ms}$ in women, were older and showed increased WBC, BUN, creatinine level and lower levels of $\mathrm{Ca}$ and albumin, and more severe patients compared with normal ECG group.

\section{DISCUSSION}

Orientia tsutsugamushi is known to spread systemically through the vascular and lymphatic systems, ${ }^{1}$ although the mechanism of infection dissemination remains unclear. In a recent murine model study, in which $O$. tsutsugamushi was injected into the right hind foot pads of mice and bacterial loads 
TABLE 3

A comparison of clinical features, laboratory findings, and ECG abnormalities between the health-care checkup group and scrub typhus patients

\begin{tabular}{|c|c|c|c|}
\hline & Health-care checkup group $(n=165)$ & Scrub typhus patient group $(n=165)$ & $P$ value \\
\hline \multicolumn{4}{|l|}{ Demographic characteristics } \\
\hline Age (years) & $63.33 \pm 13$ & $63.33 \pm 13$ & 1.000 \\
\hline Male & $58(35.2 \%)$ & $58(35.2 \%)$ & 1.000 \\
\hline \multicolumn{4}{|l|}{ Laboratory finding } \\
\hline WBC (/L) & $6,024 \pm 1,912$ & $8,241 \pm 4,036$ & $<0.001$ \\
\hline Platelets $\left(\times 10^{6} / \mathrm{L}\right)$ & $261 \pm 64.8$ & $170 \pm 91$ & $<0.001$ \\
\hline $\mathrm{Hb}(\mathrm{g} / \mathrm{dL})$ & $13.6 \pm 1.2$ & $12.6 \pm 1.7$ & $<0.001$ \\
\hline Aspartate transaminase (U/L) & $26.3 \pm 12$ & $160 \pm 460$ & $<0.001$ \\
\hline Alanine transaminase (U/L) & $23 \pm 15.2$ & $92 \pm 108$ & $<0.001$ \\
\hline Total bilirubin (mg/dL) & $0.78 \pm 0.27$ & $0.92 \pm 0.62$ & 0.008 \\
\hline blood urea nitrogen (mg/dL) & $15 \pm 4.0$ & $18 \pm 12.9$ & 0.004 \\
\hline Creatinine (mg/dL) & $1.00 \pm 0.16$ & $1.14 \pm 0.64$ & 0.006 \\
\hline ECG abnormality & $23(13.9 \%)$ & 72 (43.6\%) & $<0.001$ \\
\hline Arrhythmic group & $21(12.7 \%)$ & $31(18.8 \%)$ & 0.131 \\
\hline Ischemic change group & $13(7.9 \%)$ & $25(15.2 \%)$ & 0.039 \\
\hline Prolonged QT group & $4(2.4 \%)$ & 32 (19.4\%) & $<0.001$ \\
\hline
\end{tabular}

were subsequently measured in each organ, after the lung, the second highest bacterial load was observed in the heart, suggesting that heart tissues may experience a greater level of O. tsutsugamushi bacterial invasion than other tissues. ${ }^{7}$

In this study, groups of scrub typhus patients were compared according to the presence or absence of ECG changes on hospital admission, which were used as indicators to assess cardiac involvement and disease severity. Abnormal ECG findings at admission were observed in $43.6 \%$ of patients with scrub typhus, including $19.4 \%, 15.1 \%$, and $12.7 \%$ of patients classified as prolonged QT group, ischemic change group and arrhythmia group, respectively. Abnormal ECG in scrub typhus patients was associated with old age and increased WBC, BUN, creatinine and total bilirubin. In addition, when compared with 1:1 age and gender-matched normal subject ECG abnormalities were much more common among scrub typhus patients (43.6\% versus $13.9 \%)$. In another Thailand study, abnormal ECG was found in 39 of 79 patients

TABLE 4

A comparison of demographic and clinical characteristics, laboratory findings, and disease severity between scrub typhus patients in the normal ECG group and abnormal ECG group

\begin{tabular}{|c|c|c|c|}
\hline & Normal ECG group $(n=93)$ & Abnormal ECG group $(n=72)$ & $P$ value \\
\hline \multicolumn{4}{|l|}{ Characteristics } \\
\hline Age (years) & $61.1 \pm 13.2$ & $66.2 \pm 12.4$ & 0.014 \\
\hline Male (\%) & $29(31.2)$ & $29(40.3)$ & 0.225 \\
\hline \multicolumn{4}{|l|}{ Underlying diseases } \\
\hline Smoking (\%) & $10(10.7)$ & $9(12.5)$ & 0.736 \\
\hline Hypertension (\%) & $25(26.9)$ & $31(43.1)$ & 0.03 \\
\hline Diabetes (\%) & $13(14.0)$ & $18(25.0)$ & 0.072 \\
\hline Cardiovascular diseases (\%) & $2(2.2)$ & $2(2.8)$ & 0.795 \\
\hline Lung diseases (\%) & $2(2.2)$ & $1(1.4)$ & 0.716 \\
\hline Hepatic diseases (\%) & $7(7.5)$ & $7(9.7)$ & 0.795 \\
\hline Cancer (\%) & $4(4.3)$ & $4(5.6)$ & 0.710 \\
\hline \multicolumn{4}{|l|}{ Laboratory } \\
\hline White blood cell (/L) & $7,457 \pm 3,430$ & $9,253 \pm 4,531$ & 0.006 \\
\hline Platelets $\left(\times 10^{6} / \mathrm{L}\right)$ & $173 \pm 104$ & $167 \pm 70$ & 0.659 \\
\hline Erythrocyte sedimentation rate (mm/Hour) & $21.8 \pm 19.3$ & $23.8 \pm 19$ & 0.517 \\
\hline C-reactive protein (mg/dL) & $8.3 \pm 8$ & $10.1 \pm 7.3$ & 0.153 \\
\hline Alkaline phosphatase (U/L) & $106 \pm 70$ & $114 \pm 101$ & 0.572 \\
\hline Aspartate transaminase $(U / L)$ & $170 \pm 553$ & $147 \pm 303$ & 0.745 \\
\hline Alanine transaminase (U/L) & $92 \pm 108$ & $91 \pm 107$ & 0.955 \\
\hline Albumin (g/dL) & $3.6 \pm 0.6$ & $3.5 \pm 0.6$ & 0.216 \\
\hline Total bilirubin (mg/dL) & $0.8 \pm 0.53$ & $1.06 \pm 0.7$ & 0.012 \\
\hline Blood urea nitrogen (mg/dL) & $14.9 \pm 9.3$ & $22.3 \pm 15.5$ & 0.001 \\
\hline Creatinine (mg/dL) & $1.02 \pm 0.42$ & $1.31 \pm 0.82$ & 0.007 \\
\hline CK-MB (ng/mL) & $5.18 \pm 6.5$ & $10.3 \pm 36.4$ & 0.289 \\
\hline Troponin-T (ng/mL) & $0.014 \pm 0.13$ & $0.016 \pm 0.13$ & 0.447 \\
\hline $\mathrm{N}$-terminal probrain natriuretic peptide $(\mathrm{pg} / \mathrm{mL})$ & $150 \pm 46$ & $3,397 \pm 3,793$ & 0.115 \\
\hline $\mathrm{Na}(\mathrm{mEq} / \mathrm{L})$ & $137 \pm 4.7$ & $136 \pm 4.3$ & 0.073 \\
\hline $\mathrm{K}(\mathrm{mEq} / \mathrm{L})$ & $3.9 \pm 0.47$ & $3.9 \pm 0.53$ & 0.802 \\
\hline $\mathrm{Cl}(\mathrm{mEq} / \mathrm{L})$ & $104 \pm 4.8$ & $103 \pm 5.7$ & 0.139 \\
\hline Lactate dehydrogenase (U/L) & $610 \pm 316$ & $685 \pm 488$ & 0.255 \\
\hline $\mathrm{Ca}(\mathrm{mg} / \mathrm{dL})$ & $8.18 \pm 0.53$ & $8.00 \pm 0.63$ & 0.141 \\
\hline $\mathrm{Mg}(\mathrm{mg} / \mathrm{dL})$ & $2.09 \pm 0.38$ & $2.07 \pm 0.38$ & 0.850 \\
\hline Phosphate (mg/dL) & $3.07 \pm 1.06$ & $3.24 \pm 1.16$ & 0.490 \\
\hline Severe scrub tyhpus (\%) & $19(20.4)$ & $25(34.7)$ & 0.04 \\
\hline
\end{tabular}


TABLE 5

A comparison of demographic and clinical characteristics, laboratory findings and disease severity between scrub typhus patients in the normal ECG group and abnormal ECG subgroup

\begin{tabular}{|c|c|c|c|c|c|}
\hline & \multirow{2}{*}{$\frac{\text { Normal ECG group }}{(n=93)}$} & \multicolumn{4}{|c|}{ Abnormal ECG Subgroup } \\
\hline & & Prolonged QT $(n=32)$ & $P$ value & Arrhythmia $(n=31)$ & $P$ value \\
\hline \multicolumn{6}{|l|}{ Characteristics } \\
\hline Age (years) & $61.1 \pm 13.2$ & $67.6 \pm 11.9$ & 0.016 & $67.5 \pm 12.3$ & 0.019 \\
\hline Male (\%) & 29 (31.2) & $15(46.9)$ & 0.109 & $17(54.8)$ & 0.018 \\
\hline \multicolumn{6}{|l|}{ Underlying diseases } \\
\hline Smoking (\%) & $10(10.7)$ & $3(9.4)$ & 0.859 & $4(12.9)$ & 0.956 \\
\hline Hypertension (\%) & $25(26.9)$ & $12(37.5)$ & 0.256 & $16(51.6)$ & 0.011 \\
\hline Diabetes (\%) & $13(14.0)$ & $8(25.0)$ & 0.150 & $10(32.3)$ & 0.023 \\
\hline Cardiovascular diseases (\%) & $2(2.2)$ & $2(6.3)$ & 0.256 & $1(3.2)$ & 0.736 \\
\hline Lung diseases (\%) & $2(2.2)$ & $1(3.1)$ & 0.756 & $0(0.0)$ & 0.410 \\
\hline Hepatic diseases (\%) & $7(7.5)$ & $4(12.5)$ & 0.475 & $3(9.7)$ & 0.912 \\
\hline Cancer (\%) & $4(4.3)$ & $1(3.1)$ & 0.770 & $2(6.5)$ & 0.629 \\
\hline \multicolumn{6}{|l|}{ Laboratory } \\
\hline White blood cell (/L) & $7,457 \pm 3,430$ & $10,388 \pm 4,874$ & 0.001 & $9,930 \pm 5,336$ & 0.02 \\
\hline Platelets $\left(\times 10^{6} / \mathrm{L}\right)$ & $173 \pm 104$ & $184 \pm 67$ & 0.583 & $159 \pm 74$ & 0.496 \\
\hline Erythrocyte sedimentation rate (mm/Hour) & $21.8 \pm 19.3$ & $25.2 \pm 20.4$ & 0.403 & $25.6 \pm 20.4$ & 0.368 \\
\hline C-reactive protein (mg/dL) & $8.3 \pm 8$ & $10.9 \pm 7.6$ & 0.113 & $11.5 \pm 6.4$ & 0.053 \\
\hline Alkaline phosphatase (U/L) & $106 \pm 70$ & $119 \pm 112$ & 0.477 & $105 \pm 96.7$ & 0.936 \\
\hline Aspartate transaminase (U/L) & $170 \pm 553$ & $180 \pm 440$ & 0.930 & $139 \pm 146$ & 0.756 \\
\hline Alanine transaminase (U/L) & $92 \pm 108$ & $89 \pm 127$ & 0.894 & $97 \pm 101$ & 0.830 \\
\hline Albumin (g/dL) & $3.6 \pm 0.6$ & $3.4 \pm 0.6$ & 0.044 & $3.4 \pm 0.6$ & 0.055 \\
\hline T-bilirubin (mg/dL) & $0.8 \pm 0.53$ & $1.02 \pm 0.57$ & 0.057 & $1.06 \pm 0.57$ & 0.026 \\
\hline Blood urea nitrogen (mg/dL) & $14.9 \pm 9.3$ & $24.7 \pm 17.5$ & 0.004 & $22.5 \pm 14.6$ & 0.009 \\
\hline Creatinine (mg/dL) & $1.02 \pm 0.42$ & $1.41 \pm 1.06$ & 0.048 & $1.24 \pm 0.49$ & 0.014 \\
\hline CK-MB (ng/mL) & $5.18 \pm 6.5$ & $14.4 \pm 51$ & 0.348 & $7.39 \pm 14.5$ & 0.307 \\
\hline Troponin-T (ng/mL) & $0.014 \pm 0.13$ & $0.016 \pm 0.014$ & 0.466 & $0.016 \pm 0.015$ & 0.414 \\
\hline $\mathrm{N}$-terminal probrain natriuretic peptide (pg/mL) & $150 \pm 46$ & $4,106 \pm 4,733$ & 0.05 & $4,980 \pm 5,189$ & 0.109 \\
\hline $\mathrm{Na}(\mathrm{mEq} / \mathrm{L})$ & $137 \pm 4.7$ & $136 \pm 3.9$ & 0.130 & $134 \pm 4.3$ & 0.001 \\
\hline $\mathrm{K}(\mathrm{mEq} / \mathrm{L})$ & $3.9 \pm 0.47$ & $3.95 \pm 0.53$ & 0.877 & $4.0 \pm 0.59$ & 0.548 \\
\hline $\mathrm{Cl}(\mathrm{mEq} / \mathrm{L})$ & $104 \pm 4.8$ & $103 \pm 5.5$ & 0.247 & $102 \pm 5.6$ & 0.013 \\
\hline Lactate dehydrogenase (U/L) & $610 \pm 316$ & $726 \pm 594$ & 0.181 & $752 \pm 417$ & 0.063 \\
\hline $\mathrm{Ca}(\mathrm{mg} / \mathrm{dL})$ & $8.18 \pm 0.53$ & $7.86 \pm 0.55$ & 0.038 & $7.87 \pm 0.45$ & 0.022 \\
\hline $\mathrm{Mg}(\mathrm{mg} / \mathrm{dL})$ & $2.09 \pm 0.38$ & $2.12 \pm 0.38$ & 0.739 & $2.07 \pm 0.35$ & 0.897 \\
\hline Phosphate (mg/dL) & $3.07 \pm 1.06$ & $3.39 \pm 1.38$ & 0.329 & $3.11 \pm 1.16$ & 0.886 \\
\hline Severe scrub tyhpus (\%) & $19(20.4)$ & 13 (40.6) & 0.024 & $8(25.8)$ & 0.530 \\
\hline
\end{tabular}

(49.4\%), including sinus tachycardia(17 patients, $21.5 \%)$, new onset AFib (nine patients, $11.3 \%){ }^{8}$

In a Papua New Guinea study conducted in 1945, no significant ECG changes were observed in patients with scrub typhus ${ }^{9}$; a study conducted in Thailand in 2002 revealed that mild ECG changes (e.g., ST-T change, U wave, VPB) were observed in only $24 \%$ of patients. ${ }^{10}$ However, in this study, ECG abnormalities were observed in 72 (43.6\%) of 165 scrub typhus patients and this result is higher than previous report by Kim et al., ${ }^{11}$ where ECG abnormalities were observed in about one-third of scrub typhus patients. Therefore, ECG abnormalities were observed in a considerable number of scrub typhus patients in this study compared with previous studies. Whether this difference in frequency is due to differences in virulence related to the $O$. tsutsugamushi genotype or serotype or to differences in racial sensitivity is unclear. The subjects of the study conducted in Papua New Guinea [9] were mostly military personnel and thus likely comprised relatively young men, although their ages were not indicated. Moreover, as the mean age of patients with scrub typhus in the study from Thailand was 39 years [10], influences related to age or underlying disease would likely explain the observed differences in the frequency of ECG abnormalities. ${ }^{12}$

Atrial fibrillation was the most commonly observed type of arrhythmia observed in patients with scrub typhus. In Korea, the reported prevalence rates of Afib were $0.7 \%$ in people aged $\geq 40$ years, $2.1 \%$ in people aged $\geq 65$ years, and $4.0 \%$ in people aged $\geq 80$ years. ${ }^{13}$ In this study, the health checkup group had a mean age of 63 years and showed an $1.8 \%$ Afib prevalence, whereas the scrub typhus patients group showed a higher Afib prevalence of $9.1 \%(P=0.004)$ despite a lack of difference in age.

The mechanism by which ECG changes occur in patients with scrub typhus has not yet been clearly determined. However, various cardiac complications, such as myocarditis, ${ }^{14}$ myocardial infarction, ${ }^{15}$ and pericarditis ${ }^{16}$ have been reported in patients with scrub typhus. Orientia tsutsugamushi may proliferate and induce apoptosis in the microvascular endothelium, leading to vasculitis and perivasculitis in multiple organs systemically. Particularly, the most remarkable change occurs in the interventricular septum of the heart, and both focal and general myocarditis have been reported. However, because involvement of the coronary artery and heart valve is very rare and no severe cardiac myofibril damage has been observed, chronic heart damage is known to rarely occur, similar to other rickettsial diseases. ${ }^{17}$

It is possible that ECG abnormalities in patients with scrub typhus may be caused by an electrolyte imbalance or fever, in addition to cardiac abnormalities. Woodward et al. ${ }^{18}$ reported that low-voltage QRS abnormalities and Afib on ECG might be suggestive of myocardial damage. However, a prospective 
study conducted in Taiwan found no differences in QRS wave abnormalities between patients with scrub typhus and normal subjects; this study also showed that 17 and 14 of 27 patients with scrub typhus had low serum potassium concentrations during the febrile and recovery periods, respectively, suggesting a possible relationship between hypokalemia and ECG abnormalities. ${ }^{10}$ Furthermore, a study by Kim et al. found high BUN in patients with arrhythmia at admission and high BUN and creatinine levels in patients with abnormalities during follow-up ECG, suggesting that an electrolyte imbalance or increased creatinine level is likely to be associated with ECG changes. ${ }^{11}$ In the present study, BUN and creatinine levels were significantly higher in patients with abnormal ECG findings, whereas serum sodium (134.3 mEq/L versus $137.3 \mathrm{mEq} / \mathrm{L}$, $P=0.008)$ and calcium levels $(7.84 \mathrm{mg} / \mathrm{dL}$ versus $8.18 \mathrm{mg} / \mathrm{dL}$, $P=0.023$ ) were low in patients with scrub typhus in the arrhythmia group. In particular, the most common ECG abnormality, QT prolongation, is known to be induced by various drugs or electrolyte abnormalities (e.g., hypokalemia, hypomagnesemia, and hypocalcemia) and can cause severe ventricular arrhythmias, such as torsade de pointes. $5,19,20$ Surprisingly, the finding of this study suggesting that QT prolongation was most prevalent is very important clinically. Torsade de pointes, a life-threatening arrhythmia, was reported to have occurred in patients with scrub typhus receiving azithromycin. ${ }^{21}$ If patients with QT prolongation are given macrolide or quinolone antibiotics, which can cause QT prolongation, ventricular arrhythmia may occur; therefore, caution is required when using these aforementioned drugs. In addition to drugs, QT prolongation may be induced by an electrolyte imbalance. Among scrub typhus patients, the prolonged QT group showed lower total calcium $(7.86 \mathrm{mg} / \mathrm{dL}$ versus $8.18 \mathrm{mg} / \mathrm{dL}, P=0.038$ ) and ionized calcium levels than the normal ECG group (4.09 $\mathrm{mg} / \mathrm{dL}$ versus $4.27 \mathrm{mg} / \mathrm{dL}, P=0.018$ ). Therefore, we believe that systematic studies of the causes of hypocalcemia in patients with scrub typhus, as well as the relationship with ECG abnormalities such as QT prolongation, are needed in the future.

Although the symptoms used to assess severity in patients treated for scrub typhus include hypotension, thrombocytopenia, leukocytosis, acute kidney injury, hypoalbuminemia, and hepatic injury, the initial ECG findings are often overlooked. A study by Kim et al. ${ }^{11}$ suggested that initial ECG finding abnormalities might be related to uremia or admission into the intensive care unit and, accordingly, that ECG findings at the time of patient admission should be carefully checked, as continued follow-up is required if an ECG abnormality is found. In the present study, ECG abnormalities at admission were implicated in disease severity, especially in prolonged QT group, although there was no significant difference in arrhythmic group. So an ECG abnormality is considered a possible manifestation of severe disease.

The present study had certain limitations, including its retrospective study design and single-institution setting. The second, many patients were transferred from other hospitals for failure to respond to treatment or comorbidities, and it may therefore be difficult to generalize the subjects of this study to the entire patient population. The third, for most cases, preadmission ECG findings were unknown, and in many cases, it was difficult to accurately determine whether the abnormal ECG findings at admission were actually associated with scrub typhus. The fourth, the use of QTc prolongation agents in all patients included in this study was not fully investigated because it is retrospective study. However, almost all possible scrub typhus patients included in this study were initially checked whether the antibiotics such as macrolide, quinolone were not administered before admitted to Chosun University Hospital or not.

Nevertheless, this was the first study to compare the frequencies of different types of ECG abnormalities between patients with scrub typhus and controls on a large scale. This study demonstrated that ECG abnormalities were more commonly observed in patients with scrub typhus than in the control health checkup group. In addition, patients with scrub typhus who presented with prolonged QT interval at admission showed greater disease severity than normal ECG group. Thus, clinicians must pay more attention when administering antibiotics that may prolong QT interval.

Received July 15, 2017. Accepted for publication October 8, 2018.

Published online December 10, 2018.

Acknowledgments: This study was supported by a research fund from Chosun University Hospital, 2016.

Authors' addresses: Seo-Won Choi, Na Ra Yun, Dong-Hyun Choi, Young-Jae Ki, and Dong-Min Kim, Internal medicine, Chosun University, College of Medicine, Gwangju, Republic of Korea, E-mails: mdswchoi@gmail.com, shine@chosun.ac.kr, dhchoi@chosun.ac.kr, bonds0427@naver.com, and drongkim@chosun.ac.kr. Seok Won Kim, Department of Neurosurgery, Chosun University College of Medicine, Gwangju, Republic of Korea, E-mail: chosunns@chosun. ac.kr. Choon-Mee Kim, Premedical Science, College of Medicine, Gwangju, Republic of Korea, E-mail: choonmee@chosun.ac.kr.

\section{REFERENCES}

1. Elisberg BL, Campbell JM, Bozeman FM, 1968. Antigenic diversity of rickettsia tsutsugamushi: epidemiologic and ecologic significance. J Hyg Epidemiol Microbiol Immunol 12: 18-25.

2. Jensenius M, Fournier PE, Raoult D, 2004. Rickettsioses and the international traveler. Clin Infect Dis 39: 1493-1499.

3. Jensenius M, Montelius R, Berild D, Vene S, 2006. Scrub typhus imported to Scandinavia. Scand J Infect Dis 38: 200-202.

4. Cracco C, Delafosse C, Baril L, Lefort Y, Morelot C, Derenne JP, Bricaire F, Similowski T, 2000. Multiple organ failure complicating probable scrub typhus. Clin Infect Dis 31: 191-192.

5. Tsay RW, Chang FY, 1998. Serious complications in scrub typhus. J Microbiol Immunol Infect 31: 240-244.

6. Blackburn H, Keys A, Simonson E, Rautaharju P, Punsar S, 1960. The electrocardiogram in population studies. A classification system. Circulation 21: 1160-1175.

7. Keller CA, Hauptmann M, Kolbaum J, Gharaibeh M, Neumann M, Glatzel M, Fleischer B, 2014. Dissemination of Orientia tsutsugamushi and inflammatory responses in a murine model of scrub typhus. PLoS Negl Trop Dis 8: e3064.

8. Thipmontree W, Tantibhedhyangkul W, Silpasakorn S, Wongsawat E, Waywa D, Suputtamongkol Y, 2016. Scrub typhus in northeastern Thailand: eschar distribution, abnormal electrocardiographic findings, and predictors of fatal outcome. Am J Trop Med Hyg 95: 769-773.

9. Howell WL, 1945. Absence of electrocardiographic changes in Tsutsugamushi fever (scrub typhus); report of two hundred consecutive cases. Arch Intern Med (Chic) 76: 217.

10. Watt $G$, Kantipong $P$, Jirajarus $K, 2002$. Acute scrub typhus in Northern Thailand: EKG changes. Southeast Asian J Trop Med Public Health 33: 312-313.

11. Kim JM, Hyun M, Kim HA, Kim MK, Kim JY, Kwon KY, Ryu SY, 2014. A review of the electrocardiography changes and 
clinical manifestations of scrub typhus in a single center. Korean J Med 86: 301-307.

12. Hong SP, Kim DM, 2014. A review of electrocardiography changes and clinical manifestation in scrub typhus in a single center. J Korean Internal Med 86: 298-300.

13. Son MK, Lim NK, Cho MC, Park HY, 2016. Incidence and risk factors for atrial fibrillation in Korea: the national health insurance service database (2002-2010). Korean Circ J 46: $515-521$.

14. Sittiwangkul $R$, Pongprot $Y$, Silviliarat $S$, Oberdorfer $P$, Jittamala $P$, Sirisanthana $V, 2008$. Acute fulminant myocarditis in scrub typhus. Ann Trop Paediatr 28: 149-154.

15. Kim DG, Kim JW, Choi YS, Kim SH, Kim SM, Park CG, Seo HS, Oh DJ, 2007. Acute myocardial infarction following scrub typhus infection. Int J Cardiol 114: e18-e20.

16. Levine HD, 1946. Pathologic study of thirty-one cases of scrub typhus fever with especial reference to the cardiovascular system. Am Heart J 31: 314-328.
17. Irons EM, Armstrong HE, 1947. Scrub typhus in Dutch New Guinea. Ann Intern Med 26: 201-220.

18. Woodward TE, Mc CF Jr., Carey TN, Togo Y, 1960. Viral and rickettsial causes of cardiac disease, including the coxsackie virus etiology of pericarditis and myocarditis. Ann Intem Med 53:1130-1150.

19. McGowan GK, Walters G, 1976. Ventricular arrhythmias and hypokalemia. Lancet 2: 964.

20. Mineoi K, Matsuoka $H$, Sumimoto $T$, Kawada $H$, Hamada $M$, Hiwada K, Kondoh T, Ochi T, 1992. Torsade de pointes induced by hypocalcemia in a postoperative patient with thyrotoxicosis. Jpn Heart J 33: 735-738.

21. Lim JH, Kim ES, Chung MH, Kang JS, 2008. Torsades de pointes by azithromycin in scrub typhus and review on cardiac manifestations of scrub typhus. Infect Chemother 40: 58-62.

22. Prineas RJ, Crow RS, Zhang ZM, 2010. The Minnesota Code Manual of Electrocardiographic Findings, Standards and Procedures for Measurement and Classification. 2nd edn. London, UK: Springer. 